\title{
Trace formulas for stochastic evolution operators: Weak noise perturbation theory
}

\author{
Predrag Cvitanović, C.P. Dettmann, Ronnie Mainieri, and Gábor Vattay \\ Center for Chaos and Turbulence Studies \\ Niels Bohr Institute \\ Blegdamsvej 17, DK-2100 Copenhagen Ø
}

July 16, 2018

PACS: 02.50.Ey, 03.20.+i, 03.65.Sq, 05.40.+j, 05.45.+b

keywords: noise, stochastic dynamics, cycle expansions, semiclassical limit, periodic orbits, Perron-Frobenius operator, spectral determinant, zeta functions.

\begin{abstract}
Periodic orbit theory is an effective tool for the analysis of classical and quantum chaotic systems. In this paper we extend this approach to stochastic systems, in particular to mappings with additive noise. The theory is cast in the standard field theoretic formalism, and weak noise perturbation theory written in terms of Feynman diagrams. The result is a stochastic analog of the next-to-leading $\hbar$ corrections to the Gutzwiller trace formula, with long time averages calculated from periodic orbits of the deterministic system. The perturbative corrections are computed analytically and tested numerically on a simple 1-dimensional system.
\end{abstract}

\section{Introduction}

Noise plays important role in a variety of physical contexts. Robustness to noise is of interest for any system since there is always some small length scale at which the dynamics is affected by thermal or quantum fluctuations or unobserved degrees of freedom. For example, the interplay of deterministic dynamics and magnetic diffusivity is subject of great interest in the dynamo problem, where the effect of magnetic field diffusion on the steady fast kinematic dynamo rates is discussed in [1] within the periodic orbit theory formulation of refs. [2, 3, 4]. 
The noise tends to regularize the theory, replacing the deterministic delta function evolution operators by smooth distributions. While in this paper we are interested in effects of weak but finite noise, the $\sigma \rightarrow 0$ limit is also important as a tool for identifying the natural measure [5, 6, 6] for deterministic flows. The noise regularization might in addition cure some of the ills of intermittent systems which are plagued by power-law convergences arising from terms like $|\Lambda-1|^{-1}$ in the limit $\Lambda \rightarrow 1$.

We have cast the theory in the standard field theoretic language [8], in the spirit of approaches such as the Martin-Siggia-Rose [9] formalism, the Parisi$\mathrm{Wu}$ [10] stochastic quantization, and the Feigenbaum and Hasslacher [11] study of noise renormalization in period doubling. This perturbation theory has the same structure as the $\hbar$ corrections to the semiclassical Gutzwiller trace formulas [12] computed by Gaspard and Alonso [13, 14, 15], and the trace formulas for continuous stochastic flows and for the $\hbar$ corrections formulated by Vattay [16].

Though it is clear from the literature on stochastic path integrals that some kind of Feynman diagrams apply, the present work seems to be one of the few that actually compute the weak noise corrections for a concrete dynamical system, although in some cases the leading correction may be obtained directly from the perturbed eigenfunction [17, 18]. The form of the perturbative expansions of sect. 1 is reminiscent of perturbative calculations of field thery, but in some aspects the calculations undertaken here are relatively more difficult. The main difference is that there is no translational invariance along the chain, so unlike the case of usual field theory, the propagator is not diagonalized by a Fourier transform. We do our computations in configuration coordinates. Unlike the most field-theoretic literature, we are neither "quantizing" around a trivial vacuum, nor a countable infinity of stable soliton saddles, but around an infinity of nontrivial unstable hyperbolic saddles.

Two aspects of our results are a priori far from obvious: (a) that the structure of the periodic orbit theory should survive introduction of noise, and (b) a more subtle and surprising result, repeats of prime cycles can be resummed and theory reduced to the dynamical zeta functions and spectral determinants of the same form as the for the deterministic systems.

Having constructed the perturbation expansion in sect. 1, in sect. 5 we confront the theory with a numerical determination of eigenfunctions and eigenvalues, and verify the correctness of our perturbation expansion to the same numerical accuracy. A variety of flow models with noise are simpler to study in nonperturbative (large $\sigma$ ) limits; numerical eigenfunctions do not depend on the weak noise assumptions, and in fact require the noise to be larger than the effective discretization length of the basis. 


\section{Stochastic evolution operator}

The periodic orbit theory allows us to calculate long time averages in a chaotic system as expansions in terms of the periodic orbits (cycles) of the system. The simplest example is provided by the Perron-Frobenius operator

$$
\mathcal{L} \rho(y)=\int d x \delta(y-f(x)) \rho(x)
$$

for a deterministic map $f(x)$ which maps a density distribution $\rho(x)$ forward in time. The periodic orbit theory relates the spectrum of this operator and its weighted evolution operator generalizations to the periodic orbits via trace formulas, dynamical zeta functions and spectral determinants [19, 20]. Our purpose here is to develop the parallel theory for stochastic dynamics, given by the discrete Langevin equation [21, 22]

$$
x_{n+1}=f\left(x_{n}\right)+\sigma \xi_{n},
$$

where the $\xi_{n}$ are independent normalized Gaussian random variables.

We shall treat a chaotic system with such Gaussian weak external noise by replacing the the deterministic evolution $\delta$-function kernel by $\mathcal{L}_{\sigma}$, the FokkerPlanck kernel corresponding to (1), a sharply peaked noise distribution function

$$
\mathcal{L}_{\sigma}=\delta_{\sigma}(y-f(x))
$$

where $\delta_{\sigma}$ is the Gaussian kernel

$$
\delta_{\sigma}(z)=\frac{1}{\sqrt{2 \pi \sigma^{2}}} e^{-z^{2} / 2 \sigma^{2}}
$$

The method can be applied to smooth distributions other than the Gaussian one in the same manner.

We shall evaluate the trace formulas by steepest descent methods, and obtain the noisy traces (traces of $\mathcal{L}_{\sigma}$ ) and determinants in terms of the cycles of the deterministic system. The theory is then tested numerically on one-dimensional maps, but we expect the generalization to higher dimensions to be of the same structure as the formulas derived here. 


\section{Stochastic trace formula, steepest descent approxi- mation}

We start by calculating the trace of the $n$th iterate of the stochastic evolution operator $\mathcal{L}_{\sigma}$ for a one-dimensional analytic map $f(x)$ with additive Gaussian noise $\sigma$. This trace is an $n$-dimensional integral on $n$ points along a discrete periodic chain, so $x$ becomes an $n$-vector $x_{a}$ with indices $a, b, \ldots$ ranging from 0 to $n-1$ in a cyclic fashion

$$
\begin{aligned}
\operatorname{tr} \mathcal{L}_{\sigma}^{n} & =\int[d x] \exp \left\{-\frac{1}{2 \sigma^{2}} \sum_{a}\left[x_{a+1}-f\left(x_{a}\right)\right]^{2}\right\} \\
x_{n} & =x_{0}, \quad[d x]=\prod_{a=0}^{n-1} \frac{d x_{a}}{\sqrt{2 \pi \sigma^{2}}} .
\end{aligned}
$$

As we are dealing with a path integral on a finite discrete chain, we find it convenient to rewrite the exponent in matrix notation

$$
\operatorname{tr} \mathcal{L}_{\sigma}^{n}=\int[d x] e^{-\left[h^{-1} x-f(x)\right]^{2} / 2 \sigma^{2}}, \quad h_{a b}=\delta_{a, b+1}
$$

where $x$ and $f(x)$ are column vectors with components $x_{a}$ and $f\left(x_{a}\right)$ respectively, and $h$ is the left cyclic shift or hopping matrix satisfying $h^{n}=1, h^{-1}=h^{T}$. Unless stated otherwise, we shall assume the repeated index summation convention throughout, and that the Kronecker $\delta$ function is the periodic one, defined by

$$
\delta_{a b}=\frac{1}{n} \sum_{k=0}^{n-1} e^{i 2 \pi(a-b) k / n}
$$

For sufficiently short chains, (4) is an integral that conceivably lends itself to numerical evaluation 23], although clearly not in the long time $n \rightarrow \infty$ limit. However, if the noise is weak, the path integral (国) is dominated by periodic deterministic trajectories. Assuming that the periodic points of given finite period $n$ are isolated and the trajectory broadening $\sigma$ sufficiently small so that they remain clearly separated, the dominant contributions come from neighborhoods of periodic points; in the saddlepoint approximation the trace (4) is given by

$$
\operatorname{tr} \mathcal{L}_{\sigma}^{n} \longrightarrow \sum_{x_{c} \in \mathrm{Fix}^{n}} e^{W_{c}}
$$


where the sum goes over all periodic points $x_{c}=x_{c+n}$ of period $n, f^{n}\left(x_{c}\right)=x_{c}$. The contribution of the $x_{c}$ neighborhood is obtained by shifting the origin of integration to

$$
x_{a} \rightarrow x_{a}+\phi_{a}
$$

where from now on $x_{a}$ refers to the position of the $a$-th periodic point, and expanding $f$ in Taylor series around each of the periodic points in the orbit of $x_{c}$.

The contribution of the neighborhood of the periodic point $x_{c}$ is given by

$$
\begin{aligned}
e^{W_{c}} & =\int[d \phi] e^{-\left(\Delta^{-1} \phi-V^{\prime}(\phi)\right)^{2} / 2 \sigma^{2}} \\
& =|\operatorname{det} \Delta| \int[d \varphi] e^{\sum \frac{1}{k} \operatorname{tr}\left(\Delta V^{\prime \prime}(\phi)\right)^{k}} e^{-\varphi^{2} / 2 \sigma^{2}}
\end{aligned}
$$

where the propagator and interaction terms are collected in

$$
\Delta_{a b}^{-1} \phi_{b}=-f^{\prime}\left(x_{a}\right) \phi_{a}+\phi_{a+1}, \quad V(\phi)=\sum_{a} \sum_{m=2}^{\infty} f^{(m)}\left(x_{a}\right) \frac{\phi_{a}^{m+1}}{(m+1) !}
$$

We find it convenient to also introduce a bidirectional propagator $C=\Delta \Delta^{T}$ for reasons that will become apparent below. In the second line of (8) we have changed coordinates,

$$
\varphi=\Delta^{-1} \phi-V^{\prime}(\phi)
$$

and used the matrix identity $\ln \operatorname{det} M=\operatorname{tr} \ln M$ on the Jacobian

$$
\frac{1}{\operatorname{det}\left(\Delta^{-1}-V^{\prime \prime}\right)}=\frac{\operatorname{det} \Delta}{\operatorname{det}\left(1-\Delta V^{\prime \prime}\right)}=\operatorname{det} \Delta e^{-\operatorname{tr} \ln \left(1-\Delta V^{\prime \prime}\right)}
$$

The functional dependence of $\phi=\phi(\varphi)$ is recovered by iterating (10)

$$
\phi_{a}=\Delta_{a b} \varphi_{b}+\Delta_{a b} V_{b}^{\prime}(\phi)
$$

The above manipulations are standard [9] and often used in the "stochastic quantization" literature [10, 24], where they are artfully employed to promote identities such as $\operatorname{det} M / \operatorname{det} M=1$ to supersymmetric field theories. Such symmetries do not seem to simplify the calculation at hand. 
The saddlepoint expansion is most conveniently evaluated in terms of Feynmann diagrams, which we now introduce. The interaction terms in $V$ and its derivatives can be represented in terms of the vertices

$$
f^{\prime \prime}\left(x_{a}\right)=\longrightarrow, f^{\prime \prime \prime}\left(x_{a}\right)=\longrightarrow
$$

and the propagators as directed lines

$$
\begin{aligned}
& \Delta_{a b}=\longrightarrow \\
& C_{a b}=\longrightarrow
\end{aligned}
$$

The first two derivatives of $V$ may be written

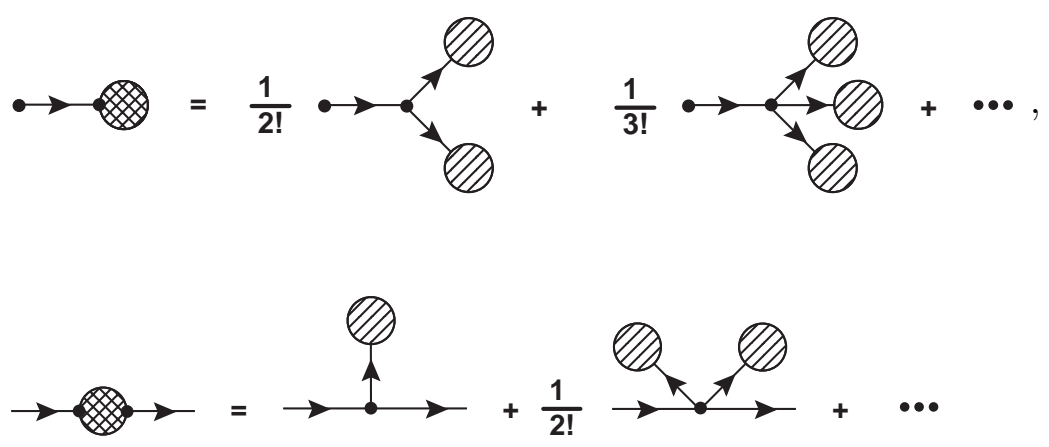

with the cross-hatched circle as $V$ and the diagonally filled circle as $\phi$. The relation between the fields (12) becomes

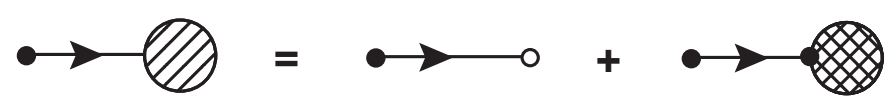

with the small open circle as $\varphi$. This recursively generates all tree diagrams ending in $\varphi$, which the stochastic averaging of sect. 4 will tie into loop corrections.

As the sum is cyclic, $e^{W_{c}}$ is the same for all periodic points in a given cycle, independent of the choice of the starting point $x_{c}$.

In the saddlepoint approximation we assume that the map is analytic and the extrema $f^{n}$ are isolated. For the leading $\sigma^{2}$ correction that we shall evaluate 
here we need derivatives of $f$ up to the third. A map with non-analytic points or marginal stability would lead to additional diffraction corrections that we shall not consider here.

From the second path integral representation in (8) it follows that $\Delta$ can be interpreted as the "free" propagator. As $\Delta$ will play a central role in what follows, we write its inverse in its full $[n \times n]$ matrix form:

$$
\Delta^{-1}=h^{-1}-\mathbf{f}^{\prime}=\left(\begin{array}{ccccc}
-f_{0}^{\prime} & 1 & & & \\
& -f_{1}^{\prime} & 1 & & \\
& & -f_{2}^{\prime} & 1 & \\
& & & \ddots & \\
1 & & & & -f_{n-1}^{\prime}
\end{array}\right)
$$

where $\mathbf{f}^{\prime}$ is a diagonal matrix with elements $f_{a}^{\prime}=f^{\prime}\left(x_{a}\right)$ a shorthand notation for stability of the map at the periodic point $x_{a}$. The determinant of $\Delta$ is

$$
\operatorname{det} \Delta=\frac{(-1)^{n}}{\Lambda_{c}-1}, \quad \Lambda_{c}=\prod_{a=0}^{n-1} f^{\prime}\left(x_{a}\right)
$$

with $\Lambda_{c}$ the stability of the $n$ cycle going through the periodic point $x_{c}$. We shall assume that we are dealing with a chaotic dynamical system, and that all cycles are unstable, $\left|\Lambda_{c}\right|>1$.

The formula for propagator itself is obtained by inverting (16) and using relation $\left(h \mathbf{f}^{\prime}\right)^{n}=\Lambda_{c}$, (due to the periodicity of the chain):

$$
\begin{aligned}
\Delta & =-\frac{1}{1-\mathbf{f}^{\prime-1} h^{-1}} \mathbf{f}^{\prime-1}=-\sum_{k=0}^{\infty}\left(\mathbf{f}^{\prime-1} h^{-1}\right)^{k} \mathbf{f}^{\prime-1} \\
& =-\frac{1}{\Lambda_{c}-1} \sum_{k=0}^{n-1} h\left(\mathbf{f}^{\prime} h\right)^{k}
\end{aligned}
$$

In the full matrix form, the propagator is given by

$$
\Delta=\frac{-1}{\Lambda_{c}-1}\left(\begin{array}{cccccc}
f_{1}^{\prime} \ldots f_{n-1}^{\prime} & f_{2}^{\prime} \ldots f_{n-1}^{\prime} & f_{3}^{\prime} \ldots f_{n-1}^{\prime} & & \ldots & 1 \\
1 & f_{2}^{\prime} \ldots f_{0}^{\prime} & f_{3}^{\prime} f_{4}^{\prime} \ldots f_{0}^{\prime} & & \ldots & f_{0}^{\prime} \\
f_{1}^{\prime} & 1 & f_{3}^{\prime} \ldots f_{0}^{\prime} f_{1}^{\prime} & & \ldots & f_{0}^{\prime} f_{1}^{\prime} \\
f_{1}^{\prime} f_{2}^{\prime} & f_{2}^{\prime} & 1 & \ddots & & f_{0}^{\prime} f_{1}^{\prime} f_{2}^{\prime} \\
f_{1}^{\prime} f_{2}^{\prime} f_{3}^{\prime} & f_{2}^{\prime} f_{3}^{\prime} & f_{3}^{\prime} & & \ddots & \vdots \\
\vdots & \vdots & \vdots & \vdots & & \vdots \\
f_{1}^{\prime} \ldots f_{n-2}^{\prime} & f_{2}^{\prime} \ldots f_{n-2}^{\prime} & \ldots & \ldots & 1 & f_{0}^{\prime} \ldots f_{n-2}^{\prime}
\end{array}\right)
$$


or, more compactly,

$$
\Delta_{a b}=\frac{-1}{\Lambda_{c}-1} \prod_{d=b+1}^{a-1} f^{\prime}\left(x_{d}\right), \quad \Delta_{a, a-1}=\frac{-1}{\Lambda_{c}-1}
$$

where $d$ increases cyclically through the range $b+1$ to $a-1$; for example, if $a=0, a-1=n-1$. We note that $\Delta$ is invertible only for cycles which are not marginal, $\left|\Lambda_{c}\right| \neq 1$. The $\left|\Lambda_{c}\right|=1$ case we would require going beyond the Gaussian saddlepoints studied here, and typically to the Airy-function type stationary points [25].

\section{Weak noise perturbation expansion}

The saddlepoint approximation (8) is a discrete path integral on periodic chain of $n$ points which we shall evaluate by standard field-theoretic methods. Separating the quadratic terms we obtain

$$
e^{W_{c}}=\frac{1}{\left|\Lambda_{c}-1\right|} \int[d \varphi] e^{-S_{0}(\varphi)-S_{I}(\varphi)}
$$

where

$$
S_{0}(\varphi)=\varphi^{2} / 2 \sigma^{2}, \quad S_{I}(\varphi)=-\sum_{k=1}^{\infty} \frac{1}{k} \operatorname{tr}\left[\Delta V^{\prime \prime}(\phi(\varphi))\right]^{k}
$$

The terms collected in $S_{I}(\varphi)$, linear or higher in $\varphi$, are the interaction vertices.

Next introduce a source term $J_{a}$ and define a partition function

$$
\begin{aligned}
e^{W_{c}(J)} & =\frac{1}{\left|\Lambda_{c}-1\right|} \int[d \varphi] e^{-S_{0}(\varphi)-S_{I}(\varphi)+J_{a} \varphi_{a}} \\
& =\frac{1}{\left|\Lambda_{c}-1\right|} e^{-S_{I}\left(\frac{d}{d J}\right)} \int[d \varphi] e^{-S_{0}(\varphi)+J_{a} \varphi_{a}} \\
& =\frac{1}{\left|\Lambda_{c}-1\right|} e^{-S_{I}\left(\frac{d}{d J}\right)} e^{\frac{\sigma^{2}}{2} J^{2}}
\end{aligned}
$$

Here we have used standard formulas for Gaussian integrals together with the normalization (四). In our diagrammatic notation this is

$$
\left.\mathrm{e}^{\mathrm{W}_{\mathrm{c}}[\mathrm{J}]}=\frac{1}{\left|\Lambda_{\mathrm{c}}-1\right|} \mathrm{e}^{\left\{\bigcirc+\frac{1}{2}\right.}+\cdots\right\} \mathrm{e}^{\frac{\sigma^{2}}{2} \longleftrightarrow \mathrm{x}}
$$


Expanding

$$
e^{\frac{\sigma^{2}}{2} J^{2}}=1+\frac{\sigma^{2}}{2} J_{a} J_{a}+\frac{\sigma^{4}}{8} J_{a} J_{a} J_{b} J_{b}+\ldots
$$

operating on this series with $\exp \left\{-S_{I}\left(\frac{d}{d J}\right)\right\}$,

$$
\left.\frac{d}{d J_{a}} \frac{d}{d J_{b}} e^{\frac{\sigma^{2}}{2} J_{d} J_{d}}\right|_{J=0}=\sigma^{2} \delta_{a b}, \quad \cdots,
$$

collecting terms of the same order in $\sigma^{2}$, and setting $J_{a}$ to zero yields the perturbation expansion

$$
W_{c}=-\ln \left|\Lambda_{c}-1\right|+\sum_{k=1}^{\infty} W_{c, 2 k} \sigma^{2 k}
$$

In field-theoretic calculations the $W_{c, 0}$ term is usually an overall volume term that drops out in the expectation value computations. In contrast, here the $W_{c, 0}=-\ln \left|\Lambda_{c}-1\right|$ term is the classical weight of the cycle which plays the key role both in the classical and stochastic trace formulas.

In diagrammatic language, we join all possible pairs of $\varphi$ vertices, each one giving a $\sigma^{2} C$ propagator. Thus the first diagram in (24) is expanded (14, 15) to

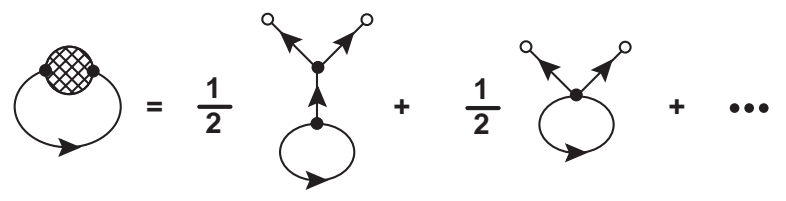

and then the $\varphi$ vertices joined to form two diagrams contributing at order $\sigma^{2}$. The full noise corrections of order $\sigma^{2}$ are given by all connected two-loop diagrams:

$$
W_{c, 2}=\frac{1}{2} \bigcirc \rightarrow \circlearrowleft+\frac{1}{2} \bigcirc \rightarrow
$$

Each diagram has a two-fold symmetry, hence all combinatorial weights equal $1 / 2$. Before writing down the final expression, we note that several sub-diagrams may be simplified using (20). These are (no sum on $a, b$ )

$$
\begin{aligned}
& \bigcirc_{\mathrm{a}}=\Delta_{a a}=\frac{-\Lambda_{c}}{\Lambda_{c}-1} \frac{1}{f_{a}^{\prime}} \\
& \mathrm{b}_{\mathrm{b}}=\Delta_{a b} \Delta_{b a}=\frac{\Lambda_{c}}{\left(\Lambda_{c}-1\right)^{2}} \frac{1+\left(\Lambda_{c}-1\right) \delta_{a b}}{f_{a}^{\prime} f_{b}^{\prime}} .
\end{aligned}
$$


Adding the terms we obtain the $\sigma^{2}$ contribution to the trace:

$$
\begin{aligned}
W_{c, 2}=\frac{1}{2}[ & \frac{\Lambda_{c}}{\Lambda_{c}-1} \sum_{a}\left(\frac{f_{a}^{\prime \prime 2}}{f_{a}^{\prime 2}}-\frac{f_{a}^{\prime \prime \prime}}{f_{a}^{\prime}}\right) C_{a a} \\
& \left.+\frac{\Lambda_{c}^{2}+\Lambda_{c}}{\left(\Lambda_{c}-1\right)^{2}} \sum_{a b} \frac{f_{a}^{\prime \prime}}{f_{a}^{\prime}} \frac{f_{b}^{\prime \prime}}{f_{b}^{\prime}} C_{a b}-\frac{\Lambda_{c}}{\Lambda_{c}-1} \sum_{a b} \frac{f_{a}^{\prime \prime}}{f_{a}^{\prime}} f_{b}^{\prime \prime} \Delta_{a b} C_{b b}\right]
\end{aligned}
$$

For an alternative approach to evaluating multiple derivatives, see appendix A.

\subsection{Repeats of prime cycles}

In the deterministic case repeats of periodic orbits can be summed up, and spectral determinants and dynamical zeta functions written in terms of prime cycles rather than periodic points. In order to accomplish this for the stochastic case, we need to compute the trace for repeats of periodic orbits.

For $r$ repeats of a prime cycle $p$ we have $n=n_{p} r, \Lambda_{c}=\Lambda_{p}^{r}$, where $\Lambda_{p}$ is the stability of the prime cycle $p$. Each index $a=0, \ldots, n-1$ is decomposed as $a=\dot{a}+\bar{a} n_{p}+\hat{a}$ with $\dot{a}=0, \ldots, n_{p}-1$ and $\bar{a}=0, \ldots, r-1$. $\hat{a}$ is an arbitrary starting point on the orbit which may be chosen independently for each index. $f$ and its derivatives depend only on $\dot{a}$.

The first sum in (29) is

$$
\frac{1}{2} \sum_{\dot{a} \bar{a} \dot{b} \bar{b}} \frac{\Lambda_{p}^{r}}{\left(\Lambda_{p}^{r}-1\right)^{3}}\left(\frac{f_{\dot{a}}^{\prime \prime 2}}{f_{\dot{a}}^{\prime 2}}-\frac{f_{\dot{a}}^{\prime \prime \prime}}{f_{\dot{a}}^{\prime}}\right) \Lambda_{p}^{2(\bar{a}-\bar{b})} \prod_{\dot{d}=\dot{b}+1}^{\dot{a}-1} f_{\dot{d}}^{\prime 2}
$$

where $\bar{a}-\bar{b}$ is the number of full repeats of $p$ contained in $\prod_{d=b+1}^{a-1}$; this is achieved by setting $\hat{a}=b+1$. The sums over $\bar{a}$ and $\bar{b}$ are performed, leading to

$$
\frac{r}{2} \frac{\Lambda_{p}^{2 r}-1}{\Lambda_{p}^{2}-1} \frac{\Lambda_{p}^{r}}{\left(\Lambda_{p}^{r}-1\right)^{3}} \sum_{\dot{a} \dot{b}}\left(\frac{f_{\dot{a}}^{\prime \prime 2}}{f_{\dot{a}}^{\prime 2}}-\frac{f_{\dot{a}}^{\prime \prime \prime}}{f_{\dot{a}}^{\prime}}\right) \prod_{\dot{d}=\dot{b}+1}^{\dot{a}-1} f_{\dot{d}}^{\prime 2}
$$

which is just a factor depending on $r$ and $\Lambda_{p}$ multiplied by the sum for the single repeat of the prime cycle.

When the calculations are carried out for both of the other sums, some rather unenlightening algebra leads to exactly the same prefactor; we discuss this rather remarkable point and its generalization to higher orders in detail in the sequel 
paper [26]. Combining all three terms of (29) leads to an expression for the trace in terms of cycles:

$$
\begin{aligned}
\operatorname{tr} \frac{z \mathcal{L}_{\sigma}}{1-z \mathcal{L}_{\sigma}} & =\sum_{n=1}^{\infty} z^{n} \sum_{x_{c} \in \operatorname{Fix} f^{n}} e^{W_{c}} \\
& =\sum_{p} n_{p} \sum_{r=1}^{\infty} \frac{z^{n_{p} r}}{\left|\Lambda_{p}^{r}-1\right|} \exp \left\{\frac{\sigma^{2}}{2} w_{p, 2} \frac{\Lambda_{p}^{r}\left(\Lambda_{p}^{r}+1\right)}{\left(\Lambda_{p}^{r}-1\right)^{2}} r\right\}
\end{aligned}
$$

up to order $\sigma^{2}$, where

$$
\begin{aligned}
w_{p, 2}= & \frac{\Lambda_{p}-1}{\Lambda_{p}+1} \sum_{a}\left(\frac{f_{a}^{\prime \prime 2}}{f_{a}^{\prime 2}}-\frac{f_{a}^{\prime \prime \prime}}{f_{a}^{\prime}}\right) C_{a a} \\
& +\sum_{a b} \frac{f_{a}^{\prime \prime}}{f_{a}^{\prime}} \frac{f_{b}^{\prime \prime}}{f_{b}^{\prime}} C_{a b}-\frac{\Lambda_{p}-1}{\Lambda_{p}+1} \sum_{a b} \frac{f_{a}^{\prime \prime}}{f_{a}^{\prime}} f_{b}^{\prime \prime} \Delta_{a b} C_{b b}
\end{aligned}
$$

contains all the dependence on the higher derivatives along the prime cycle $p$, with no dependence on the repetition number $r$. To put it another way, if $p$ is a cycle, not necessarily prime, then

$$
w_{p^{r}, 2}=r w_{p, 2}
$$

Next, using the identity

$$
\frac{1+x}{(1-x)^{3}}=\sum_{k=0}^{\infty}(k+1)^{2} x^{k}
$$

we rewrite the trace formula in a form in which repeats are resummed over by expanding the exponential in (30) to order $\sigma^{2}$, forming the sum over $k$, and putting the result back in an exponential:

$$
\begin{aligned}
\operatorname{tr} \frac{z \mathcal{L}_{\sigma}}{1-z \mathcal{L}_{\sigma}} & =\sum_{p} n_{p} \sum_{k=0}^{\infty} \sum_{r=1}^{\infty} \frac{z^{n_{p} r}}{\left|\Lambda_{p}^{r}\right| \Lambda_{p}^{k r}} e^{r \frac{\sigma^{2}}{2}(k+1)^{2} w_{p, 2}}+O\left(\sigma^{4}\right) \\
& =\sum_{p} n_{p} \sum_{k=0}^{\infty} \frac{t_{p, k}}{1-t_{p, k}}
\end{aligned}
$$

where $t_{p, k}$ is the $k$-th local eigenvalue

$$
t_{p, k}=\frac{z^{n_{p}}}{\left|\Lambda_{p}\right| \Lambda_{p}^{k}} e^{\frac{\sigma^{2}}{2}(k+1)^{2} w_{p, 2}}+O\left(\sigma^{4}\right)
$$


This is the stochastic equivalent of the Gutzwiller trace formula for the semiclassical case 12].

We sum over $r$ as usual [20] to obtain from (33) a Selberg type product for the noisy spectral determinant

$$
\operatorname{det}\left(1-z \mathcal{L}_{\sigma}\right)=\prod_{p} \prod_{k=0}^{\infty}\left(1-t_{p, k}\right)
$$

valid to order $\sigma^{2}$.

We observe a crossover effect, since for higher order eigenvalues (large $k$ ), eventually the argument of the exponential becomes of order one, and further noise corrections are required. This is as it should be: the higher order eigenfunctions have more detailed structure, are more quickly smeared by the noise, and should decay faster.

\section{$4.2 \quad$ Fixed point}

The contribution from a fixed point (cycle of length one) is particularly simple, as all the sums and products collapse to a single term, and $f^{\prime}=\Lambda$. We obtain

$$
w_{p, 2}=\frac{1}{\Lambda(\Lambda+1)}\left\{3\left(\frac{f^{\prime \prime}}{\Lambda-1}\right)^{2}-\frac{f^{\prime \prime \prime}}{\Lambda-1}\right\}
$$

If the map contains only a single isolated unstable fixed point, we thus have an expression for the eigenvalues,

$$
\nu_{k}(\sigma)=\frac{1}{z_{k}(\sigma)}=\frac{1}{|\Lambda| \Lambda^{k}} e^{\frac{\sigma^{2}}{2}(k+1)^{2} w_{p, 2}}
$$

valid to order $\sigma^{2}$. Note that depending on the sign of $w_{p, 2}$, small amounts of noise can either enhance or inhibit escape from the fixed point. Higher order terms for a fixed point are given in ref. [26].

\section{$5 \quad$ Numerical tests}

To test the above expressions for the trace, we have computed the required derivatives for the 23 prime cycles up to length $n=6$ for the quartic map

$$
f(x)=20\left(\frac{1}{2^{4}}-\left(\frac{1}{2}-x\right)^{4}\right)
$$


The choice of the map is motivated by requiring that the system be simple (onedimensional in this case), with non-trivial $f^{\prime \prime}, f^{\prime \prime \prime}$ (hence quartic), with complete binary dynamics (hence a nice repeller), and no diffraction and nonhyperbolic regions in the immediate vicinity of the repeller (where the Gaussian saddle points would be insufficient).

\subsection{Evaluation of the determinant}

In this we follow the approach to computing escape rates originally introduced by Kadanoff and Tang [27]. The topological length truncated cycle expansions [20] of spectral determinant (34) are obtained by writing the trace and determinant as power series expansions in $z$ and $\sigma$,

$$
\begin{aligned}
\operatorname{tr} \frac{z \mathcal{L}_{\sigma}}{1-z \mathcal{L}_{\sigma}} & =\sum_{n=1}^{N} z^{n}\left(C_{n, 0}+\sigma^{2} C_{n, 2}\right) \\
\operatorname{det}\left(1-z \mathcal{L}_{\sigma}\right) & =1-\sum_{n=1}^{N} z^{n}\left(c_{n, 0}+\sigma^{2} c_{n, 2}\right)
\end{aligned}
$$

Here, the $C$ coefficients come from (30), and the $c$ coefficients are obtained by equating coefficients in

$$
\operatorname{det}\left(1-z \mathcal{L}_{\sigma}\right) \operatorname{tr} \frac{z \mathcal{L}_{\sigma}}{1-z \mathcal{L}_{\sigma}}=-z \frac{d}{d z} \operatorname{det}\left(1-z \mathcal{L}_{\sigma}\right)
$$

following from the identity $\ln \operatorname{det} M=\operatorname{tr} \ln M$. The solution is found recursively as

$$
\begin{aligned}
c_{n, 0}= & \frac{1}{n}\left[C_{n, 0}-C_{n-1,0} c_{1,0}-\ldots-C_{1,0} c_{n-1,0}\right] \\
c_{n, 2}= & \frac{1}{n}\left[C_{n, 2}-\left(C_{n-1,0} c_{1,2}+C_{n-1,2} c_{1,0}\right)-\right. \\
& \left.\quad \ldots-\left(C_{1,0} c_{n-1,2}+C_{1,2} c_{n-1,0}\right)\right]
\end{aligned}
$$

From the $c_{n, 0}$ coefficients we construct the deterministic Fredholm determinant, from which the deterministic eigenvalue $\nu_{0}$ is found using Newton's method on the characteristic equation for $\mathcal{L}_{\sigma}$ at $\sigma=0$ :

$$
1-\sum_{n=1}^{N} \nu_{0}^{-1} c_{n, 0}=0
$$




\begin{tabular}{lll}
\hline$n$ & $\nu_{0}$ & $\nu_{0,2}$ \\
\hline 1 & 0.308 & 0.42 \\
2 & 0.37140 & 1.422 \\
3 & 0.3711096 & 1.43555 \\
4 & 0.371110995255 & 1.435811262 \\
5 & 0.371110995234863 & 1.43581124819737 \\
6 & 0.371110995234863 & 1.43581124819749 \\
\hline
\end{tabular}

Table 1: Significant digits of the leading deterministic eigenvalue and its $\sigma^{2}$ coefficient, calculated from the spectral determinant as function of the cycle truncation length $n$. Note the superexponential convergence of both $\nu_{0}$ and $\nu_{0,2}(n=6$ result is limited by the machine precision).

The $\sigma^{2}$ correction to the eigenvalue is found from the $\sigma^{2}$ terms in the characteristic equation, and comes to

$$
\nu_{2}=-\frac{\sum_{n=1}^{N} \nu_{0}^{-n} c_{n, 2}}{\sum_{n=1}^{N} n \nu_{0}^{-n-1} c_{n, 0}}
$$

The leading eigenvalue $\nu_{0}$ for the deterministic (noiseless) map and the coefficient of the $\sigma^{2}$ correction $\nu_{0,2}$, shown in table 1, demonstrate the superexponential convergence with $n$ of spectral determinant, as expected for nice hyperbolic dynamical systems.

The escape rate $\gamma$ of the repeller is calculated directly from the eigenvalue,

$$
\gamma(\sigma)=-\ln \nu(\sigma)
$$

and hence

$$
\begin{aligned}
& \gamma_{0}=-\ln \nu_{0} \\
& \gamma_{2}=-\frac{\nu_{2}}{\nu_{0}}
\end{aligned}
$$

We have also directly tested the repeat formula (32) for our cycle set.

\subsection{Discretized eigenfunction}

In order to check the perturbative calculation we evaluate the eigenvalue numerically as a function of $\sigma$ by treating the evolution operator as a matrix acting on a discretized eigenfunction. That is, we approximate $\mathcal{L}$ by a matrix $\mathcal{L}_{y_{c} x_{c}}$ where $\left(x_{c}, y_{c}\right)$ is the center of a square in the $x-y$ plane of small but finite size $\epsilon$ (with 
upper limits $\left(x_{t}, y_{t}\right)$ and lower limits $\left.\left(x_{b}, y_{b}\right)\right)$. The matrix element is obtained by assuming the distribution $\rho$ is constant across this small square:

$$
\mathcal{L}_{y_{c} x_{c}}=\epsilon^{-1} \int_{x_{b}}^{x_{t}} \int_{y_{b}}^{y_{t}} \delta_{\sigma}(y-f(x)) d x d y
$$

The integral may be approximated using just a few evaluations of the kernel with errors of a similar order to those due to the variation in $\rho$. For example

$$
\mathcal{L}_{y_{c} x_{c}}=\frac{1}{16}\left(L_{t t}+2 L_{t c}+L_{t b}+2 L_{c t}+4 L_{c c}+2 L_{c b}+L_{b t}+2 L_{b c}+L_{b b}\right)
$$

with $L_{t c}=\delta_{\sigma}\left(y_{t}-f\left(x_{c}\right)\right)$, etc. requires only four evaluations per square since the boundary points belong to more than one square. For the map at hand, the discretized evolution operator leads to six digit accuracy in the escape rate for values of $\sigma$ as low as $3 \epsilon$.

When $\sigma$ is small the matrix is very sparse, a fact which can be used to speed up the calculation. The leading eigenvalue is obtained by repeatedly evolving and rescaling an arbitrary smooth initial distribution, which then approaches the leading eigenfunction. In our case the map is expanding on the neighborhood of the asymptotic repeller, so the eigenfunctions are smooth, and the discretization procedure is stable. The numerical eigenfunctions for two values of $\sigma$ is shown in fig. 2. The eigenfunction peaks at the critical point of the map, but this has no detectable effect on the eigenvalue, as subsequent iterations send the points around the peak towards $-\infty$, away from the repeller $x \in[0,1]$. As the flow is conserved only on the infinite interval $x \in(-\infty, \infty)$, no normalizable eigenfunction exists. On any finite interval, however, the escape rate is nonzero. If no point outside the interval can return (ignoring the exponentially small tunneling probabilities), that is, if the interval encloses the repeller and the neighboring region around it determined by the magnitude of the noise, the escape rate and the leading eigenfunction (up to normalization) are independent of the interval chosen.

Subtracting the perturbative analytic terms from the numerically computed $\nu_{0}(\sigma)$,

$$
\nu_{0}(\sigma)-0.371110995234863-1.43581124819749 \sigma^{2} \sim O\left(\sigma^{4}\right)
$$

we compare with the numerically computed $\nu_{0}(\sigma)$ in fig. 1, and estimate the next term to be approximately $38 \sigma^{4}$. 


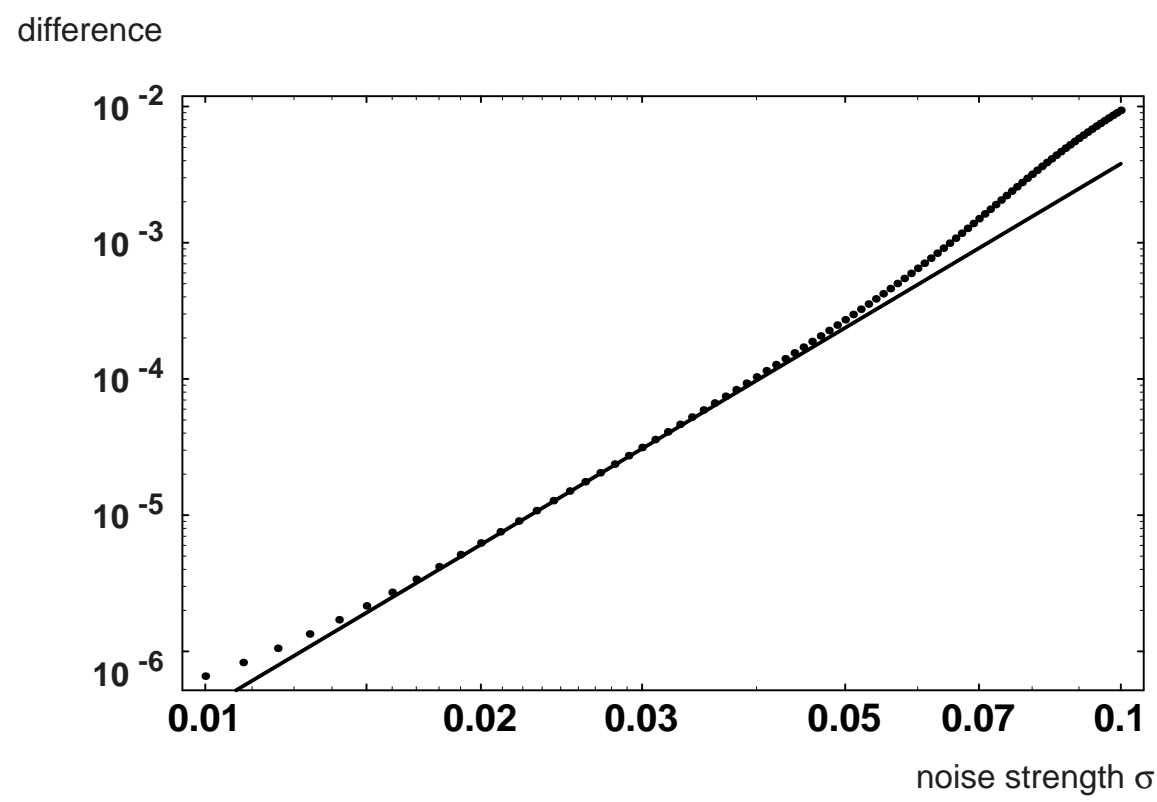

Figure 1: The deviation of the analytic estimate (44) from the numerically computed $\nu_{0}(\sigma)$ for a range of values of the noise strength $\sigma$ (points), vs. the conjectured remainder, $38 \sigma^{4}$ (solid line). For small $\sigma$ the errors are due to the finiteness of the grid, and for larger $\sigma$ the deviation is due to the neglected higher order contributions.

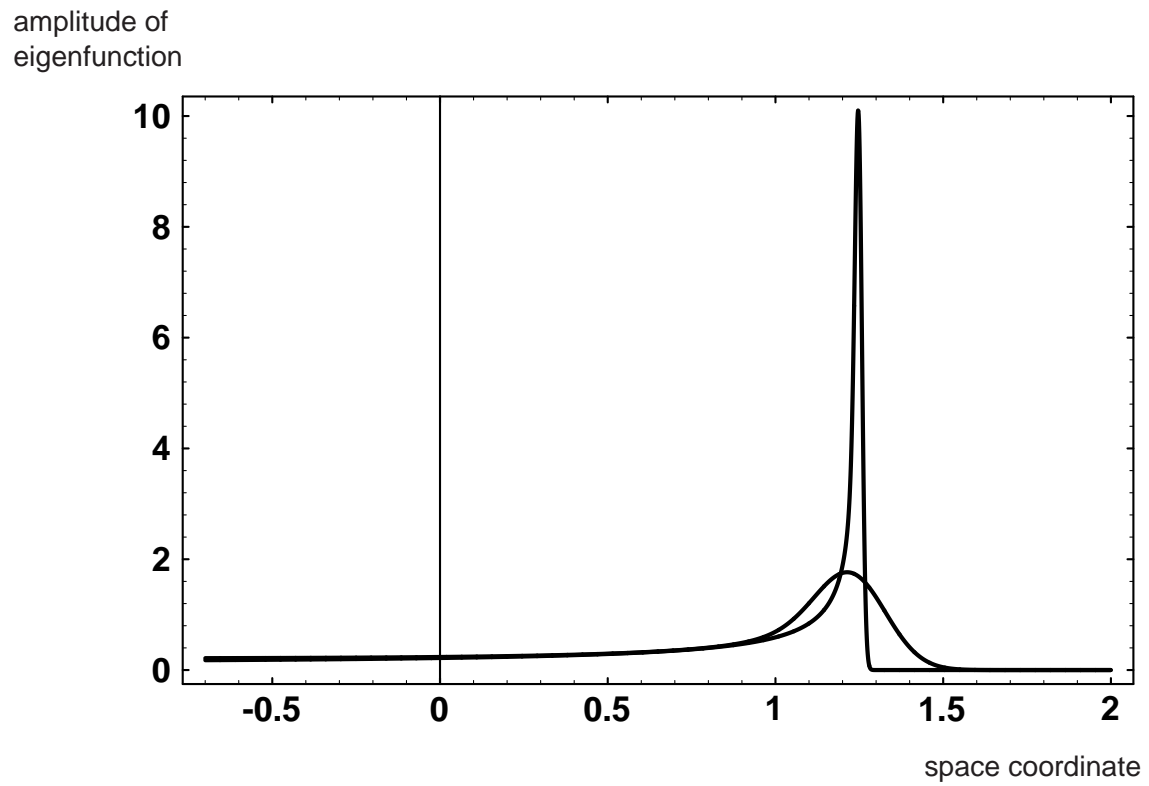

Figure 2: The eigenfunction for $\sigma=0.01$ (sharp peak) and $\sigma=0.1$ (smoother). 


\section{Summary and outlook}

We have formulated weak noise perturbation theory for noisy maps in terms of periodic orbits of the deterministic system, expanding to order $\sigma^{2}$ explicitly, resummed repeats of prime cycles, and tested the results numerically. From here, there are many possible generalizations and future directions.

In the sequel paper [26] we shall recast the remarkable resummation of repeats (sect. 4.1) in a more general framework applicable to all orders of the expansion. It seems from the numerical section that the coefficients of powers of $\sigma$ are growing very rapidly. The expansion is expected to be asymptotic.

Evaluation of expectation values [20] on a stochastic flow requires replacing the Perron-Frobenius operator (2) by the generalized evolution operator

$$
\mathcal{L}_{\sigma}=\delta_{\sigma}(y-f(x)) e^{\beta \cdot a(x)} .
$$

The same general perturbation theory applies, but now an observable $a(x)$ contributes an extra set of interaction vertices to $S_{I}(\phi)$ in (21). Similarly, the addition of more dimensions and/or non-Gaussian weak noise can be treated by modifying the propagators and adding new vertices.

While for deterministic flows it is appropriate to replace a flow by a return map on a Poincaré section of the flow, it is not clear that this is appropriate for stochastic flows; a noise that is "white" on the Langevin equation level is "colored" when integrated to a Poincaré section return, and it might have memory of the trajectory that a noisy iterated mapping cannot mimic.

The noise in general has a different structure than the deterministic equations of motion; it typically breaks whatever symmetries the classical flow might have, unless clever precautions are taken to ensure that the noise respects the symmetry [28]. This situation is familiar from Quantum Mechanics, where quantization and canonical transformations do not commute.

Our saddlepoint approximation to the spectrum of the exact evolution operator receives perturbative contributions from all cycles, no matter how long. However, the noise causes the physical system to effectively lose memory at a rate depending on the region of phase space, so it might be possible to obtain accurate averages by replacing the evolution operator by effective finite memory, finite Markov partition transfer matrices.

Such studies might enable us to understand the range of applicability of the "semi-classical" theory in greater detail than for the single cut-off time proposed in the case of semiclassical quantization by Berry and Keating [29]. Different 
regions of phase space are dominated by different time scales, and the program of periodic orbit theory allows us to use the dynamics itself, encoded in the properties of cycles, to determine at what point classical behavior is modified by semiclassical or noise corrections.

As in the semiclassical case, the saddlepoint approximation causes the multiplicative structure of the evolution operators to be lost, and one might consider extended formulation of ref. [30] to improve the analyticity of the spectral determinants. Finally, non-analytic points in the dynamics will lead to diffraction effects which are of different orders in $\sigma$, for example, the escape rate of the map $4 x(1-x)$ which has a quadratic maximum at the boundary of the deterministic repeller is of order $\sqrt{\sigma}$ [17.

\section{A Appendix: Recursive evaluation of derivatives}

The derivatives of $x_{n}=f^{n}(x)$

$$
x_{n}^{\prime}=\frac{d x_{n}}{d x}, \quad x_{n}^{\prime \prime}=\frac{d^{2} x_{n}}{d x^{2}}, \quad x_{n}^{\prime \prime \prime}=\frac{d^{3} x_{n}}{d x^{3}}, \ldots
$$

with initial values

$$
x_{0}=x, \quad \frac{d x_{0}}{d x}=1, \quad \frac{d^{2} x_{0}}{d x^{2}}=0, \quad \frac{d^{3} x_{0}}{d x^{3}}=0, \ldots
$$

can be computed recursively by

$$
\begin{aligned}
x_{n+1}^{\prime}= & f^{\prime}\left(x_{n}\right) x_{n}^{\prime}=\prod_{k=0}^{n} f^{\prime}\left(x_{k}\right) \\
x_{n+1}^{\prime \prime}= & f^{\prime \prime}\left(x_{n}\right)\left(x_{n}^{\prime}\right)^{2}+f^{\prime}\left(x_{n}\right) x_{n}^{\prime \prime}=x_{n+1}^{\prime} \sum_{k=0}^{n} \frac{f^{\prime \prime}\left(x_{k}\right)}{f^{\prime}\left(x_{k}\right)} x_{k}^{\prime} \\
x_{n+1}^{\prime \prime \prime}= & f^{\prime \prime \prime}\left(x_{n}\right)\left(x_{n}^{\prime}\right)^{3}+3 f^{\prime \prime}\left(x_{n}\right) x_{n}^{\prime} x_{n}^{\prime \prime}+f^{\prime}\left(x_{n}\right) x_{n}^{\prime \prime \prime} \\
= & x_{n+1}^{\prime} \sum_{k=0}^{n} \frac{f^{\prime \prime \prime}\left(x_{k}\right)}{f^{\prime}\left(x_{k}\right)}\left(x_{k}^{\prime}\right)^{2}+3 x_{n+1}^{\prime} \sum_{0 \leq j<k \leq n} \frac{f^{\prime \prime}\left(x_{j}\right) f^{\prime \prime}\left(x_{k}\right)}{f^{\prime}\left(x_{j}\right) f^{\prime}\left(x_{k}\right)} x_{j}^{\prime} x_{k}^{\prime} \\
x_{n+1}^{\prime \prime \prime \prime}= & f^{\prime \prime \prime \prime}\left(x_{n}\right)\left(x_{n}^{\prime}\right)^{4}+6 f^{\prime \prime \prime}\left(x_{n}\right) x_{n}^{\prime \prime}\left(x_{n}^{\prime}\right)^{2} \\
& +4 f^{\prime \prime}\left(x_{n}\right) x_{n}^{\prime \prime \prime} x_{n}^{\prime}+3 f^{\prime \prime}\left(x_{n}\right)\left(x_{n}^{\prime \prime}\right)^{2}+f^{\prime}\left(x_{n}\right) x_{n}^{\prime \prime \prime \prime},
\end{aligned}
$$

$x_{n+1}^{\prime}$ in the above has form of a propagator, $f^{\prime \prime}\left(x_{k}\right) / f^{\prime}\left(x_{k}\right) 3$-vertex, $x_{n+1}^{\prime \prime \prime}$ gets contribution from a 4-vertex diagram plus three 4-leg diagrams with two 3vertices, etc.. In another words, this iteration of the "transport equations" generates the Feynman diagram expansion. 


\section{References}

[1] P. Cvitanović and E. Ott. Steady fast kinematic dynamos: The topological entropy bound and the effect of magnetic field diffusion. In preparation.

[2] E. Aurell and A. D. Gilbert. Fast dynamos and determinants of singular intergralopertaors. Geophysical and Astrophysical Fluid Dynamics, 73:5-32, 1993.

[3] N. J. Balmforth, P. Cvitanović, G. R. Ierley, E. A. Spiegel, and G. Vattay. Advection of vector fields by chaotic flows. Annals of New York Academy of Sciences, 706:148, 1993. Volume entitled Stochastic Processes in Astrophysics.

[4] S. Childress and A. D. Gilbert. Stretch, twist, fold: the fast dynamo, volume M37 of Lecture Notes in Physics. New series M. Springer-Verlag, Berlin, 1995.

[5] Ya. G. Sinai. Gibbs measures in ergodic theory. Russian Mathematical Surveys, 166:21, 1972.

[6] R. Bowen. Equilibrium states and the ergodic theory of Anosov-diffeomorphisms, volume 470 of Lecture Notes in Mathematics. Springer-Verlag, Berlin, 1975.

[7] D. Ruelle. Thermodynamic Formalism. Addison-Wesley, Reading, 1978.

[8] P. Cvitanović. Field theory. NORDITA lecture notes, Copenhagen, January 1983. Notes prepared by Ejnar Gyldenkerne.

[9] P. C. Martin, E. D. Siggia, and H. A. Rose. Statistical mechanics of classical systems. Physical Review A, 8(1):423-437, 1973.

[10] G. Parisi and Y. Wu. Perturbation-theory without gauge fixing. Scientia Sinica, 24:483-496, 1981.

[11] M. J. Feigenbaum and B. Hasslacher. Irrational decimations and path-integrals for external noise. Physical Review Letters, 49:605-609, 1982.

[12] M. Gutzwiller. Chaos in classical and quantum mechanics. Springer-Verlag, NewYork, 1990.

[13] P. Gaspard and D. Alonso. $\hbar$ expansion for the periodic-orbit quantization of hyperbolic systems. Physical Review A, 47:R3468-R3471, 1993.

[14] P. Gaspard and D. Alonso. $\hbar$ expansion for the periodic-orbit quantization of chaotic systems. Chaos, 3:601, 1993.

[15] P. Gaspard. $\hbar$-expansion for quantum trace formulas. In G. Casati and B. Chirikov, editors, Quantum chaos between order and disorder, pages 385-404. Cambridge University Press, 1995.

[16] G. Vattay. Bohr-Sommerfeld quantization of periodic orbits. Physical Review Letters, 76:1059-1062, 1996.

[17] P. Reimann. Noisy one-dimensional maps near a crisis 1: Weak gaussian white and colored noise. Journal of Statistical Physics, 82:1467-1501, 1996.

[18] P. Reimann. Noisy one-dimensional maps near a crisis 2: General uncorrelated weak noise. Journal of Statistical Physics, 85:403-425, 1996.

[19] P. Gaspard. Chaos, Scattering and Statistical Mechanics. Cambridge Univiversity Press, Cambridge, 1997. 
[20] P. Cvitanović et al. Classical and quantum chaos: A cyclist treatise. http://www.nbi.dk/ChaosBook/, Niels Bohr Institute (Copenhagen), 1998.

[21] N. G. Van Kampen. Stochastic Processes in Physics and Chemistry. North Holland, Amsterdam, 1981.

[22] A. Lasota and M. MacKey. Chaos, Fractals, and Noise; Stochastic Aspects of Dynamics. Springer-Verlag, Berlin, 1994.

[23] C. P. Dettmann. Traces and determinants of strongly stochastic operators. chaodyn/9806019.

[24] P. Damgaard and H. Huffel, editors. Stochastic Quantization, Singapore, 1988. World Scientific.

[25] N. Bleistein and R. A. Handelsman. Asymptotic Expansions of Integrals. Dover, New York, 1986.

[26] P. Cvitanović, C. P. Dettmann, R. Mainieri, and G. Vattay. Trace formulas for stochastic evolution operators: Smooth conjugation method. In preparation.

[27] L. Kadanoff and C. Tang. Escape rate from strange repellers. Proceedings of the National Academy of Science, USA, 81:1276, 1984.

[28] O. Cepas and J. Kurchan. Canonically invariant formulation of Langevin and Fokker-Planck equations. cond-mat/9706296.

[29] M. V. Berry and J. P. Keating. A rule for quantizing chaos. Journal of Physics A, 23(21):4839-4849, 1990.

[30] P. Cvitanović and G. Vattay. Entire Fredholm determinants for evaluation of semiclassical and thermodynamical spectra. Physical Review Letters, 71:4138-4141, 1993. 